\title{
Efectos de la satisfacción y frustración de las necesidades psicológicas básicas sobre las formas de pasión por el ejercicio
}

\author{
Manuel ALCARAZ-IBÁÑEZ, Álvaro SICILIA , María J. LIROLA y \\ Rafael BURGUEÑO
}

Universidad de Almería, España

(Recibido 14 Diciembre, 2015; Aceptado 19 Abril, 2016)

RESUMEN: Basado en la teoría de la autodeterminación y en el modelo dual de pasión, el objetivo de este estudio fue determinar la influencia de la percepción de satisfacción y frustración de las necesidades psicológicas básicas en el contexto del ejercicio físico sobre las formas armoniosa (PA) y obsesiva (PO) de la pasión por esta actividad, considerando si dicha relación podría diferir en función del género. Un total de 490 estudiantes universitarios (356 hombres y 114 mujeres) de entre 17 y 44 años $\left(M_{\text {edad }}=20.49 ; D T=3.38\right)$ que manifestaron realizar ejercicio físico al menos una vez por semana, completaron un cuestionario que recogía las variables de interés. Los resultados del modelo de ecuaciones estructurales mostraron que, en general, la satisfacción y la frustración de las necesidades psicológicas básicas predecían de forma positiva la PO, mientras que tan solo la satisfacción de las necesidades predecía la PA. No obstante, se observaron diferencias en función del género en el efecto que la frustración de las necesidades psicológicas básicas ejercía sobre ambas formas de pasión. En conjunto, la satisfacción y la frustración de las necesidades psicológicas básicas explicaron un $25 \%$ y un 44\% de la varianza de la PA en hombres y mujeres, respectivamente, situándose dichos porcentajes en el $32 \%$ y el $14 \%$ en el caso de la PO. Estos resultados sugieren que, al menos en población masculina, las intervenciones destinadas a disminuir la percepción de frustración de las necesidades psicológicas básicas en el contexto del ejercicio podrían contribuir a disminuir una implicación obsesiva en esta conducta.

Palabras clave: Teoría de la autodeterminación, modelo dual de pasión, teoría de las necesidades psicológicas básicas, pasión armoniosa, pasión obsesiva.

\section{Effects of satisfaction and thwarting of basic psychological needs on the forms of passion for exercise}

ABSTRACT: Based on both self-determination theory and dualistic model of passion, the main
purpose of this study was to determine the influence of the satisfaction and thwarting of basic
psychological needs perceived in the context of exercise on the development of harmonious (HP) and
obsessive (OP) forms of passion for exercise, considering additionally if such relationship could differs
across gender. A total of 398 university students ( 256 men and 114 women), aged between 17 and 44
$\left(M_{\text {age }}=20.49 ; S D=3.38\right)$ and involved in exercise at least once a week, filled a questionnaire that
assessed the variables of interest. The results of structural equation model showed that, in general, both 
satisfaction and thwarting of basic psychological needs predicted positively OP, whereas only the satisfaction of needs predicted positively HP. Furthermore, results revealed gender differences in the effect of the thwarting of needs on both forms of passion. As a whole, both satisfaction and thwarting of needs explained $25 \%$ and $44 \%$ of the variance in HP in men and women, respectively, being those percentages $32 \%$ and $14 \%$ for OP. From a practical point of view, these results suggest that, at least in males, interventions aimed at decreasing experiences of the thwarting of needs in the exercise context could contribute to diminish an obsessive commitment to this behaviour.

Keywords: Self-determination theory; dualistic model of passion; basic psychological needs theory; harmonious passion; obsessive passion.

${ }^{1}$ Correspondencia: Álvaro Sicilia, Facultad de Ciencias de la Educación, Universidad de Almería. Carretera de Sacramento s/n, 04120 La Cañada de San Urbano (Almería), España. Tel: +34+950 015 394, Fax: +34+950 015 751. E-mail: asicilia@ual.es.

La literatura científica ofrece un conjunto de evidencias que sustentan el carácter potencialmente saludable de la práctica regular de ejercicio físico (Eime, Young, Harvey, Charity, y Payne, 2013; Hötting y Röder, 2013). No obstante, la investigación también ha mostrado que el ejercicio físico puede contribuir a la aparición de consecuencias menos deseables cuando éste se realiza de una forma obsesiva y repetitiva (Paradis, Cooke, Martin, y Hall, 2013). Un constructo que puede ayudar a entender las distintas formas que la persona tiene de implicarse con la conducta del ejercicio y sus efectos diferenciales es el de pasión.

El modelo dual de pasión (MDP; Vallerand, 2010; Vallerand et al., 2003) define la pasión como un elemento de carácter motivacional que implica una fuerte inclinación hacia una actividad que la persona ama, valora y a la cual atribuye gran importancia, de manera que invierte en ella un nivel considerable de energía y tiempo (Vallerand et al., 2003). Este modelo se nutre de los postulados de la teoría de la autodeterminación (TAD; Deci y Ryan, 2000), y en línea con éstos establece dos formas de apasionarse hacia una conducta (i.e., armoniosa y obsesiva) que derivan en consecuencias afectivas, cognitivas y conductuales muy diferentes (Vallerand, 2012).

De acuerdo con los postulados de la TAD, el ser humano tiende a estructurar e integrar en su sentido identitario de acuerdo a sus propios valores e intereses, el conjunto de elementos del entorno que le rodea, a través del proceso de “integración organísmica” (Deci y Ryan, 2000, p. 248). A su vez, el modelo dual de pasión considera como fase clave del proceso de integración el mecanismo de internalización (Vallerand, 2010; Vallerand et al., 2003) a través del cual la persona tiende a sentirse atraída por determinadas conductas, personas o cosas, de manera que persiste en su consecución o realización en función del valor atribuido a las mismas. En el caso concreto del ejercicio, el MDP plantea que el proceso de internalización vendrá condicionado por la forma en la cual la persona integra la actividad en su personalidad. Si la internalización se produce de manera completa la persona integrará la conducta de manera autónoma (i.e., de forma voluntaria y coherente con el sentido del “yo”), hecho que podría favorecer el desarrollo de una pasión armoniosa (PA) por la actividad. Por contra, si el proceso de internalización de la conducta se produce tan sólo de manera parcial, la persona integrará la actividad de manera controlada (i.e. sujeta al control de factores internos o externos ajenos a la 
propia voluntad), hecho que podría propiciar el desarrollo de una pasión obsesiva (PO) por la actividad.

Atendiendo a los postulados del MDP, la PA por el ejercicio físico reflejaría una implicación voluntaria y coherente con el sentido identitario de la persona, de manera que la práctica de esta actividad se desarrollaría en armonía con el resto de sus facetas vitales. A su vez, este tipo de implicación facilitaría la aparición de un conjunto de consecuencias afectivas, cognitivas y conductuales de carácter positivo que se extenderían en el tiempo una vez concluida la práctica de la actividad (Vallerand, 2012). Por contra, pese a que la PO por el ejercicio también implica identificarse con la actividad, valorarla, e incluso disfrutar de la misma, en este caso el deseo de involucrarse en su práctica no respondería a la propia voluntad, sino a una fuente de control surgida en respuesta a ciertas contingencias de carácter social (e.g. la necesidad de aceptación) o personal (e.g. la necesidad de ganar autoestima o de experimentar situaciones excitantes) asociadas a la actividad (Vallerand, 2010). Por tanto, la PO implica no solo que el ejercicio adquiera un rol preponderante sobre el resto de facetas vitales de la persona, sino que la actividad se instrumentalice a modo de mecanismo defensivo que, manifestado a través de una implicación de carácter rígido e inflexible, supedita el bienestar psicológico y emocional del individuo a la realización de la actividad (Vallerand, 2012).

En consonancia con los postulados de la TAD, el MDP plantea que es más probable que la persona tienda a seleccionar, valorar y adherirse a aquellas actividades en las cuales percibe que son satisfechas las necesidades psicológicas básicas de autonomía, competencia y relación (Vallerand et al., 2003). De acuerdo con la TAD (Deci y Ryan, 2000) estas tres necesidades psicológicas básicas y universales alimentan el proceso mediante el cual el individuo inicia y mantiene la conducta. La necesidad de autonomía alude a la percepción de que tanto las actividades que integran la práctica como la forma de llevarlas a cabo responden a los intereses y a la voluntad de la persona. La necesidad de competencia alude a la percepción de sentirse capaz de realizar las actividades propias de este ámbito de manera eficaz. La necesidad de relación alude a la percepción de sentirse integrado en el grupo y, por tanto, unido a las personas con las que se comparte la actividad.

No obstante, a la hora de considerar la manera en la cual la persona percibe las distintas experiencias personales en un determinado contexto, la TAD distingue entre la percepción de satisfacción y percepción de frustración de las necesidades psicológicas básicas, aludiendo este segundo caso a una situación en la que la persona considera que la posibilidad de satisfacer sus necesidades está siendo obstaculizada de forma activa (Bartholomew, Ntoumanis, Ryan, y Thøgersen-Ntoumani, 2011). En este sentido no sería lo mismo, por ejemplo, un bajo nivel de satisfacción de la necesidad de relación (e.g., sentirse poco unido a un nuevo grupo debido al poco tiempo transcurrido compartiendo la actividad) que la frustración de esta necesidad (e.g., sentirse rechazado y excluido de las distintas actividades de forma sistemática por sus compañeros). De hecho, la TAD sugiere que en respuesta a la percepción de frustración de las necesidades psicológicas básicas la persona puede desarrollar diversos mecanismos de afrontamiento, caracterizados por la pérdida del autocontrol, la adquisición de un patrón rígido de funcionamiento y la búsqueda de indicadores de valía de carácter externo, elementos todos ellos que estarían conceptualmente asociados a la definición de PO planteada por el MDP (Vallerand, 2010, 2012). 
La investigación en el contexto del ejercicio ha apoyado hasta el momento los planteamientos del MDP, confirmando una asociación positiva entre la PA y consecuencias de carácter positivo, tales como mayores niveles de bienestar psicológico (Lalande et al., 2015; Méndez-Giménez, Cecchini, y Fernández-Río, 2016; Rousseau y Vallerand, 2008), menores síntomas de malestar físico y ansiedad (Carbonneau, Vallerand, y Massicotte, 2010), o menor riesgo de lesión en el trascurso de la actividad (Stephan, Deroche, Brewer, Caudroit, y Le Scanff, 2009). Por contra, la PO por el ejercicio ha sido asociada con consecuencias de carácter negativo, tales como menores niveles de bienestar psicológico (Méndez-Giménez et al., 2016; Rousseau y Vallerand, 2008), una mayor incidencia del síndrome de burnout (Lalande et al., 2015), riesgo de adicción al ejercicio (Paradis et al., 2013), mayor presencia de situaciones de riesgo en la práctica de la actividad (Vallerand et al., 2003), o una mayor probabilidad de sufrir una lesión en el desarrollo de la misma (Stephan et al., 2009). Además, la investigación hasta el momento ha mostrado parcialmente el papel precursor de las necesidades psicológicas básicas sobre las formas de pasión. En concreto, existen evidencias empíricas que apoyan la relación positiva entre la percepción de satisfacción de las necesidades psicológicas básicas y las formas de pasión por el ejercicio (Lalande et al., 2015; Parastatidou, Doganis, Theodorakis, y Vlachopoulos, 2012). Sin embargo, el escaso número de trabajos realizados hasta la fecha no han considerado el efecto predictor de la frustración de las necesidades psicológicas básicas sobre las formas de pasión. No obstante, la relación positiva entre la frustración de las necesidades psicológicas básicas y la PO parece plausible si consideramos la similitud que a nivel teórico (Bartholomew et al., 2011; Vallerand et al., 2006) y empírico (Costa, Coppolino, y Oliva, 2015; Paradis et al., 2013) parece existir en las consecuencias asociadas a ambos constructos.

Considerando los planteamientos teóricos establecidos por la TAD y del MDP, el objetivo de este estudió fue analizar la influencia diferenciada de la percepción de satisfacción y frustración de las necesidades psicológicas básicas experimentada en el contexto del ejercicio físico sobre las formas armoniosa y obsesiva de la pasión por dicha actividad. Atendiendo tanto a los fundamentos teóricos como al conjunto de evidencias empíricas expuestas con anterioridad, se hipotetizó que mientras que la percepción de satisfacción de las necesidades psicológicas básicas predeciría de forma positiva la PA y la PO $\left(\mathrm{H}_{1}\right)$, la percepción de frustración de dichas necesidades predeciría de forma positiva la $\mathrm{PO}$, mientras que no predeciría o, en todo caso, predeciría débilmente y de forma negativa la $\mathrm{PA}\left(\mathrm{H}_{2}\right)$. Además, pese al carácter universal que la TAD otorga a la influencia de la percepción de satisfacción y frustración de las necesidades psicológicas básicas sobre las distintas consecuencias derivadas de éstas (Deci y Ryan, 2000), la investigación ha mostrado que dichos efectos podrían diferir en función del género (Weman-Josefsson, Lindwall, y Ivarsson, 2015), por lo cual las hipótesis planteadas se analizaron teniendo en cuenta las submuestras de hombres y mujeres.

\section{Método}

\section{Participantes}


Tomaron parte en el estudio 470 alumnos universitarios (356 hombres y 114 mujeres), con edades comprendidas entre 17 y 44 años $\left(M_{\text {edad }}=20.49 ; D T=3.38\right)$ y que manifestaron realizar ejercicio físico con una frecuencia de al menos una vez por semana. Para analizar la estabilidad temporal de la PES (Parastatidou et al., 2012) se utilizó una segunda muestra de 35 alumnos universitarios (25 hombres y 10 mujeres) cuyas edades se situaban entre 20 y 36 años $\left(M_{\text {edad }}=22.77 ; D T=3.40\right)$. Este grupo completó la escala en dos ocasiones, mediando dos semanas entre ambas tomas de datos.

\section{Medidas}

Satisfacción de las necesidades psicológicas en el ejercicio. Se utilizó la versión española (Sánchez y Núñez Alonso, 2007) de la Basic Psychological Needs in Exercise Scale (BPNES; Vlachopoulos y Michailidou, 2006). La escala se compone de 12 ítems, agrupados en 3 subescalas de 4 ítems que evalúan el grado de percepción de satisfacción de la necesidad de autonomía (e.g., "El programa de ejercicio físico que sigo está muy relacionado con lo que me gusta y me interesa”), competencia (e.g., "creo que el ejercicio físico es una actividad que hago bien”), y relación (e.g., "me siento muy cómodo con los otros participantes del programa de ejercicio físico”). La frase que precedió a la escala fue: “Mientras práctico ejercicio físico...”La respuesta se realizó empleando una escala tipo Likert con un rango de 1 (totalmente en desacuerdo) a 5 (totalmente de acuerdo). Mayores puntuaciones indican una mayor percepción de satisfacción de las necesidades psicológicas.

Frustración de las necesidades psicológicas básicas en el ejercicio. Se utilizó la versión adaptada al contexto español (Sicilia, Ferriz, y Sáenz-Álvarez, 2013) de la Psychological Need Thwarting Scale (PNTS; Bartholomew, Ntoumanis, Ryan, y Thøgersen-Ntoumani, 2011). Este instrumento se compone de 12 ítems agrupados en 3 factores de 4 ítems cada uno, que evalúan el grado con el que las personas perciben que están siendo frustradas sus necesidades psicológicas de autonomía (e.g. "Me siento obligado a seguir las decisiones de los demás"), competencia (e.g. "Hay situaciones donde me siento incapaz"), y relación (e.g. "Me siento rechazado por los que me rodean”) en el contexto del ejercicio físico. La frase que precedió a la escala fue: "Mientras realizo ejercicio físico...” Para la respuesta se empleó una escala Likert con un rango de 1 (totalmente en desacuerdo) a 7 (totalmente de acuerdo).

Pasión por el ejercicio. Se utilizó la versión adaptada al castellano de la PES (Parastatidou et al., 2012). El instrumento se compone de 14 ítems, agrupados en 2 factores de 7 ítems cada uno que evalúan el grado de PA (e.g. "Está en armonía con las otras actividades de mi vida") y PO (e.g. "No puedo vivir sin él”) por el ejercicio. La frase que precedió a la escala fue: "El ejercicio físico..." Para la respuesta se empleó una escala Likert con un rango de 1 (totalmente en desacuerdo) a 7 (totalmente de acuerdo).

\section{Procedimiento}

Para adaptar la PES al contexto español se empleó la estrategia de traducción inversa (Hambleton, 1996), de manera que el instrumento fue traducido en primer lugar del inglés al español, siendo posteriormente traducido al inglés por un grupo de traductores independiente al primero. Como paso previo a la administración del cuestionario éste fue cumplimentado por un 
grupo reducido de alumnos universitarios para asegurar la correcta comprensión de los ítems. Se informó a los potenciales participantes acerca de las características básicas del proyecto de investigación, reiterando el carácter voluntario y anónimo de su participación. Aquellos que manifestaron su consentimiento para participar en el estudio cumplimentaron el cuestionario en presencia de una de los autores del presente trabajo, el cual estuvo a disposición de los participantes para resolver las posibles dudas que pudieron surgir en el proceso de recogida de datos. El tiempo aproximado que los participantes emplearon para cumplimentar el cuestionario fue de 10 minutos.

\section{Análisis de Datos}

En primer lugar, dado que la PES no ha sido validada en España, se analizó la estructura factorial del instrumento mediante un análisis factorial confirmatorio (AFC). Dado que los datos no se ajustaban a los criterios de normalidad multivariada (Coeficiente de Mardia=61.35; $\mathrm{p}<$.001), el AFC se realizó empleando el método de máxima verosimilitud y una técnica bootstrapping de 5000 muestreos (Byrne, 2013). Para analizar la consistencia interna de la PES se empleó el coeficiente de fiabilidad compuesta de Raykov ( $\rho$; Raykov, 2004). Dicho coeficiente refleja la heterogeneidad de la relación de los ítems con los respectivos constructos, permitiendo integrar en su cálculo la existencia de posibles correlaciones entre los términos de error (Geldhof, Preacher, y Zyphur, 2014). Para analizar la estabilidad temporal de la PES se empleó el coeficiente de correlación intraclase (CCI) y su IC al 95\%. Se consideraron como aceptables valores de consistencia interna $(\rho)$ y de estabilidad temporal (CCI) superiores o iguales a .70 (Fleiss, 2011; Hair, Black, Babin, Anderson, y Tatham, 2006). Se estimó que los factores latentes que forman la PES ofrecían evidencias de su validez discriminante atendiendo al IC al 95\% de la correlación entre éstos, el cual no debía comprenderla unidad considerada como valor absoluto (Anderson y Gerbing, 1988). Para determinar si la estructura factorial de la PES permanecía invariante en función del género, se realizó un análisis multigrupo considerando sucesivos modelos restringidos, siguiendo el procedimiento descrito por Milfont y Fischer (2010).

Como paso previo al análisis del modelo de ecuaciones estructurales, se obtuvieron los estadísticos descriptivos y los índices de consistencia interna de las escalas utilizadas. Para determinar los posibles efectos del género en las puntuaciones de HP y OP (Marsh et al., 2013) se realizó una prueba $t$ de Student para muestras independientes en función de dicha variable.

Finalmente, se testó un modelo de ecuaciones estructurales que consideraba la influencia diferenciada de la satisfacción/frustración de las necesidades psicológicas básicas (variables independientes) sobre la pasión armoniosa/obsesiva (variables dependientes). Para determinar si las regresiones implicadas en dicho modelo diferían en función del género, se comprobó la invariancia factorial del modelo. Al igual que en anteriores trabajos (Stebbings, Taylor, Spray, y Ntoumanis, 2012) la satisfacción y la frustración de las necesidades psicológicas básicas se representaron como variables latentes formadas por las puntuaciones obtenidas en cada uno de sus respectivos factores de autonomía, competencia y relación. A su vez, las formas armoniosa y obsesiva de la pasión se representaron de acuerdo al modelo estructural obtenido en el análisis que estudió su estructura factorial (Figura 1). Observada la ausencia de normalidad multivariante (Coeficiente de Mardia= 121.20; $\mathrm{p}<.001$ ), el modelo de ecuaciones estructurales 
se estimó empleando el método de máxima verosimilitud y una técnica de bootstrapping de 5000 muestreos (Byrne, 2013).

Para juzgar la bondad del ajuste de los distintos modelos testados se utilizaron los siguientes índices de ajuste: Cociente $\chi^{2} / g l$ (chi-cuadrado/grados de libertad), CFI (Comparative Fit Index), IFI (Incremental Fit Index), RMSEA (Root Mean Square Error of Approximation) más su intervalo de confianza (IC) al 90\% y SRMS (Standardized Root Mean Square Residual). Valores de $\chi 2 / g l$ inferiores a 5 se consideraron como adecuados (Bentler, 1989). Valores de CFI e IFI superiores o cercanos a .95, y de RMSEA y SRMR inferiores o muy próximos a .06 y .08, respectivamente, se consideraron como indicativos de un excelente ajuste de los datos a los respectivos modelos (Hu y Bentler, 1999). No obstante, atendiendo a las sugerencias de autores que consideran dichos puntos de corte como excesivamente restrictivos (Marsh, Hau, y Zhonglin, 2004) se consideraron como aceptables valores de CFI e IFI iguales o superiores a .90 y de RMSEA cercanos a .08 (Browne y Cudek, 1993). Dado que el test $\chi 2$ de diferencias entre grupos se ve influenciado por el tamaño muestral, para confirmar la invariancia de los distintos modelos se estimó que la hipótesis nula debía ser rechazada en presencia de disminuciones $>.010$ en el valor de CFI en los sucesivos modelos restringidos (Chen, 2007). Se utilizó un nivel de significación $p<.05$ para los estadísticos obtenidos en los diferentes análisis, los cuales se realizaron empleando el software IBM SPSS y AMOS v.22.

\section{Resultados}

\section{Análisis Propiedades Psicométricas PSE}

Un primer AFC de la PES reveló unos índices de ajuste insatisfactorios: $\chi 2(76, N=470)$ $=485.29, p<.001 ; \chi 2 / \mathrm{gl}=6.39 ; \mathrm{CFI}=.85 ;$ IFI $=.85 ; \mathrm{RMSEA}=.107($ IC $90 \%=.098, .116) ;$ $\mathrm{SRMR}=.087$. Una inspección de los índices de modificación sugirió que permitir la correlación entre los términos de error de los ítems 8 y 9 (i.e., "No puedo vivir sin él” y "No puedo dejar de hacerlo) y los ítems 12 y 13 (i.e., "Realmente me cuesta controlar la necesidad que tengo de hacerlo" y "Tengo un sentimiento casi obsesivo hacia él”) mejoraba de forma significativa el ajuste del modelo. En el primero de los casos (i.e., ítems 8 y 9) se estimó la conveniencia de permitir esta correlación atendiendo a la similar redacción de ambos ítems (Bond et al., 2011). En el segundo de los casos (i.e., 12 y 13) esta correlación podría justificarse si se considera que una persona obsesionada por el ejercicio probablemente tenga dificultades para controlar la necesidad de realizar dicha actividad. Una vez permitidas las correlaciones entre ambos pares de ítems, los índices de ajuste del modelo (Figura 1) mejoraron ostensiblemente: $\chi 2(74, N=470)=306.28, p<.001 ; \chi 2 / g l=4.14$; CFI = .92; IFI = .92; RMSEA $=.082(\mathrm{IC} 90 \%=.072, .091)$; SRMR $=.081$. En este modelo, los pesos de regresión estandarizados fueron estadísticamente significativos ( $p<.001)$, oscilando entre .46 y .80 . 


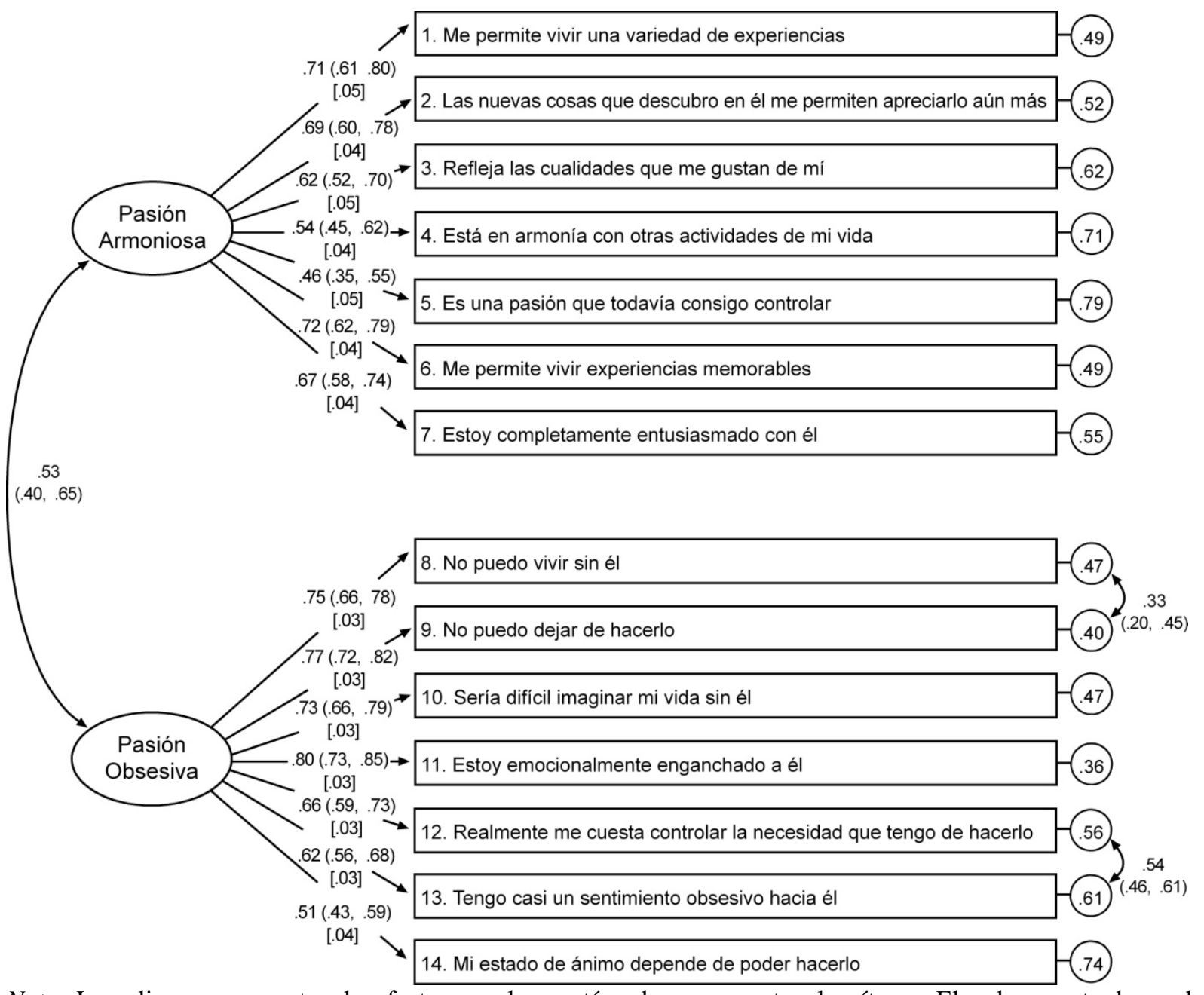

Nota. Las elipses representan los factores y los rectángulos representan los ítems. El valor mostrado en los círculos pequeños corresponde a las varianzas residuales. Los valores entre paréntesis representan el valor del IC al 95\% obtenido mediante la técnica de bootstrapping. El valor entre corchetes representa el error típico del boostrapping. La totalidad de las correlaciones y los pesos de regresión mostrados fueron estadísticamente significativos al nivel $\mathrm{p}<.01$.

Figura 1. Análisis factorial confirmatorio de la PES.

Tanto el factor PA $(\rho=.83)$ como el factor PO $(\rho=.81)$ mostraron adecuados niveles de consistencia interna. Además, ambos factores mostraron adecuados niveles de estabilidad temporal, en concreto, CCI = .91 (IC95\% .83 a .96) en el caso de la PA, y CCI= .89 (IC95\%.79 a .95) para la PO. Como se muestra en la Figura 1, el IC95\% (.40 a .61) de la correlación entre los factores latentes $(r=.53, p<.001)$ no incluyó la unidad considerada como valor absoluto, mostrando por tanto evidencias de validez discriminante para los factores que componen la PES. La Tabla 1 recoge los índices de ajuste obtenidos por la PES en el análisis multigrupo de invariancia por género. La no existencia de diferencias significativas (test $\chi^{2}$ e incrementos de CFI $\geq .01$ ) entre los sucesivos modelos restringidos, apoyó el carácter invariante en función del género de la PES. 
Tabla 1. Análisis Multigrupo de Invariancia por Género

\begin{tabular}{lcccccccccc}
\hline Modelo & $\chi^{2}$ & $g l$ & $\chi^{2} / g l$ & $\Delta \chi 2$ & $\Delta$ gl & CFI & $\Delta$ CFI & IFI & RMSEA (IC 90\%) & SRMR \\
\hline 1 & 414.13 & 148 & 2.80 & - & - & .907 & - & .908 & $.062(.055$ a .069) & .092 \\
2 & 429.17 & 160 & 2.68 & 15.04 & 12 & .906 & -0.001 & .907 & $.060(.053$ a .067$)$ & .092 \\
3 & 451.82 & 174 & 2.60 & 22.65 & 14 & .903 & -0.003 & .904 & $.058(.052$ a .065$)$ & .090 \\
4 & 484.46 & 190 & 2.55 & $32.64^{* *}$ & 16 & .898 & -0.005 & .897 & $.058(.051$ a .064$)$ & .090 \\
\hline
\end{tabular}

Nota. Modelo 1 = modelo especificado sin restricciones; Modelo 2 = modelo con pesos de medida invariantes; Modelo $3=$ modelo con pesos de medida e interceptos invariantes; Modelo 4 = modelo con pesos de medida, interceptos y residuos de medida invariantes.

${ }^{* *} \mathrm{p}<.01$

\section{Estadísticos Descriptivos}

La Tabla 2 presenta las medias, desviaciones típicas y coeficientes de consistencia interna de las escalas utilizadas. A excepción de los factores de frustración de las necesidades psicológicas básicas, las puntuaciones de ambos grupos en las distintas escalas (i.e.; hombres y mujeres) se situaron por encima del punto medio de las mismas. Dichas puntuaciones sugieren que los participantes experimentaban altos niveles de pasión y satisfacción, además de bajos niveles de frustración de sus necesidades psicológicas básicas en el contexto del ejercicio. Pese a que el valor de consistencia interna de algunas de las escalas en la muestra formada por mujeres se situó justo por debajo de .70 (i.e., entre .65 y .69), ésta puede ser considerada como aceptable atendiendo el reducido número de ítems que componían cada factor (Hair et al., 2006). La prueba $t$ de Student para muestras independientes no mostró diferencias estadísticamente significativas en las puntuaciones de PA $(t[468]=-1.385, p=.20)$ y PO $(t$ $[468]=.143, p=.15$ ) entre hombres y mujeres.

Tabla 2. Media, Desviación Típica y Fiabilidad de las Escalas para la Muestra Total, Hombres y Mujeres

\begin{tabular}{|c|c|c|c|c|c|c|c|c|c|c|}
\hline \multirow[b]{2}{*}{ Escala } & \multirow[b]{2}{*}{ Rango } & \multicolumn{3}{|c|}{$\begin{array}{c}\text { Total } \\
(N=470)\end{array}$} & \multicolumn{3}{|c|}{$\begin{array}{r}\text { Hombres } \\
(n=356)\end{array}$} & \multicolumn{3}{|c|}{$\begin{array}{l}\text { Mujeres } \\
(n=114)\end{array}$} \\
\hline & & M & DT & $\rho$ & M & DT & $\rho$ & M & DT & $\rho$ \\
\hline 1. SN Autonomía & $1-5$ & 4.31 & 0.60 & .76 & 4.28 & 0.62 & .77 & 4.40 & 0.51 & .69 \\
\hline 2. SN Competencia & $1-5$ & 4.14 & 0.60 & .74 & 4.14 & 0.61 & .73 & 4.14 & 0.59 & .77 \\
\hline 3. SN Relación & $1-5$ & 4.32 & 0.67 & .87 & 4.26 & 0.68 & .86 & 4.51 & 0.62 & 90 \\
\hline 4. FN Autonomía & $1-7$ & 1.81 & 0.93 & .76 & 1.87 & 0.98 & .78 & 1.62 & 0.75 & .65 \\
\hline 5. FN Competencia & $1-7$ & 2.24 & 1.16 & .81 & 2.22 & 1.17 & .83 & 2.29 & 1.12 & .67 \\
\hline 6. FN Relación & $1-7$ & 2.05 & 1.06 & .81 & 2.08 & 1.08 & 81 & 1.92 & 1.00 & .81 \\
\hline 7. Pasión Armoniosa & $1-7$ & 6.11 & 0.72 & .83 & 6.09 & 0.74 & .83 & 6.19 & 0.64 & .79 \\
\hline 8. Pasión Obsesiva & $1-7$ & 4.72 & 1.25 & .81 & 4.77 & 1.23 & 81 & 4.58 & 1.32 & .82 \\
\hline
\end{tabular}

Nota. $\rho=$ rho de Raykov; $\mathrm{SN}=$ satisfacción de la necesidad; $\mathrm{FN}=$ frustración de la necesidad. ${ }^{*} \mathrm{p}<.05 ;{ }^{* *} \mathrm{p}<.01$

\section{Modelos de Ecuaciones Estructurales}

El ajuste del modelo de medida se testó empleando un AFC. Tanto para la muestra total como para las submuestras de hombres y mujeres se obtuvieron adecuados valores de carga 
factorial para los indicadores de satisfacción $(>.62, p<.01)$ y frustración $(>.75, p<.01)$ de las necesidades psicológicas básicas, además de bajos errores típicos de bootstrapping (<.01). Los tres modelos estructurales testados ofrecieron aceptables índices de ajuste tanto para la muestra global como para las dos submuestras consideradas (Tabla 3). Pese a que el resultado del test de diferencias basado en el estadístico $\chi^{2}$ sugería la existencia de diferencias en el modelo de medida entre ambos grupos $(\Delta \chi 2=29.35, \Delta \mathrm{gl}=16, \mathrm{p}=.022)$, atendiendo al mínimo aumento del valor de CFI (-.002) se estimó que la estructura factorial permanecía invariante, de manera que los resultados de ambos grupos podían ser comparados.

Tabla 3. Bondad del Ajuste del Modelo Estructural para la Muestra Total $(N=470)$, Hombres $(n=356)$, y Mujeres $(n=114)$

\begin{tabular}{lccccccc}
\hline Modelo & $\chi^{2}$ & $g l$ & $\chi^{2} / g l$ & CFI & IFI & RMSEA (IC 90\%) & SRMR \\
\hline Total & 522.60 & 162 & 3.23 & .920 & .920 & $.069(.062$. a .076) & .068 \\
Hombres & 445.65 & 162 & 2.75 & .920 & .920 & $.070(.062$. a .078) & .075 \\
Mujeres & 257.68 & 162 & 1.59 & .908 & .911 & $.072(.055$. a .089) & .081 \\
\hline
\end{tabular}

El modelo estructural testado (Figura 2) mostró que la satisfacción de las necesidades psicológicas básicas predecía de forma positiva y estadísticamente significativa la PA y la PO tanto en el grupo de hombres como en el de mujeres. Por contra, se observó que, pese a no alcanzar en ambos casos el grado de significación estadística establecido, la frustración de las necesidades psicológicas básicas predecía de forma negativa la PA en hombres y de forma positiva en mujeres. A su vez, la frustración de las necesidades psicológicas básicas predecía de forma positiva y estadísticamente significativa la $\mathrm{PO}$ en hombres, mientras que, pese a no alcanzar el grado de significación estadística establecido, lo hacía de forma negativa en mujeres.

Siguiendo el procedimiento descrito por Byrne (2013), se comprobó que el modelo en el cual los pesos de regresión se fijaron al mismo valor en ambos grupos mostraba diferencias significativas respecto al modelo en el cual los pesos de regresión se estimaron libremente $(\Delta \chi 2=282.61, \Delta \mathrm{gl}=8, \mathrm{p}<.001 ; \Delta \mathrm{CFI}=.061)$. Al objeto de confirmar cuáles de las relaciones planteadas en el modelo diferían en función del género, los pesos de regresión fueron restringidos de forma secuencial e individualizada. Este análisis corroboró que el efecto de la frustración de las necesidades sobre la PA $(\Delta \chi 2=255.73, \Delta \mathrm{gl}=2, \mathrm{p}<.001 ; \Delta \mathrm{CFI}=.055)$ y la PO $(\Delta \chi 2=97.15, \Delta \mathrm{gl}=2, \mathrm{p}<.001 ; \Delta \mathrm{CFI}=.021)$ difería en ambos grupos. En su conjunto, la percepción de satisfacción y frustración de las necesidades psicológicas básicas explicó, respectivamente, un 25\% de la PA y un 32\% de la PO en hombres, y un $44 \%$ de la PA y un $14 \%$ de la PO en mujeres. 


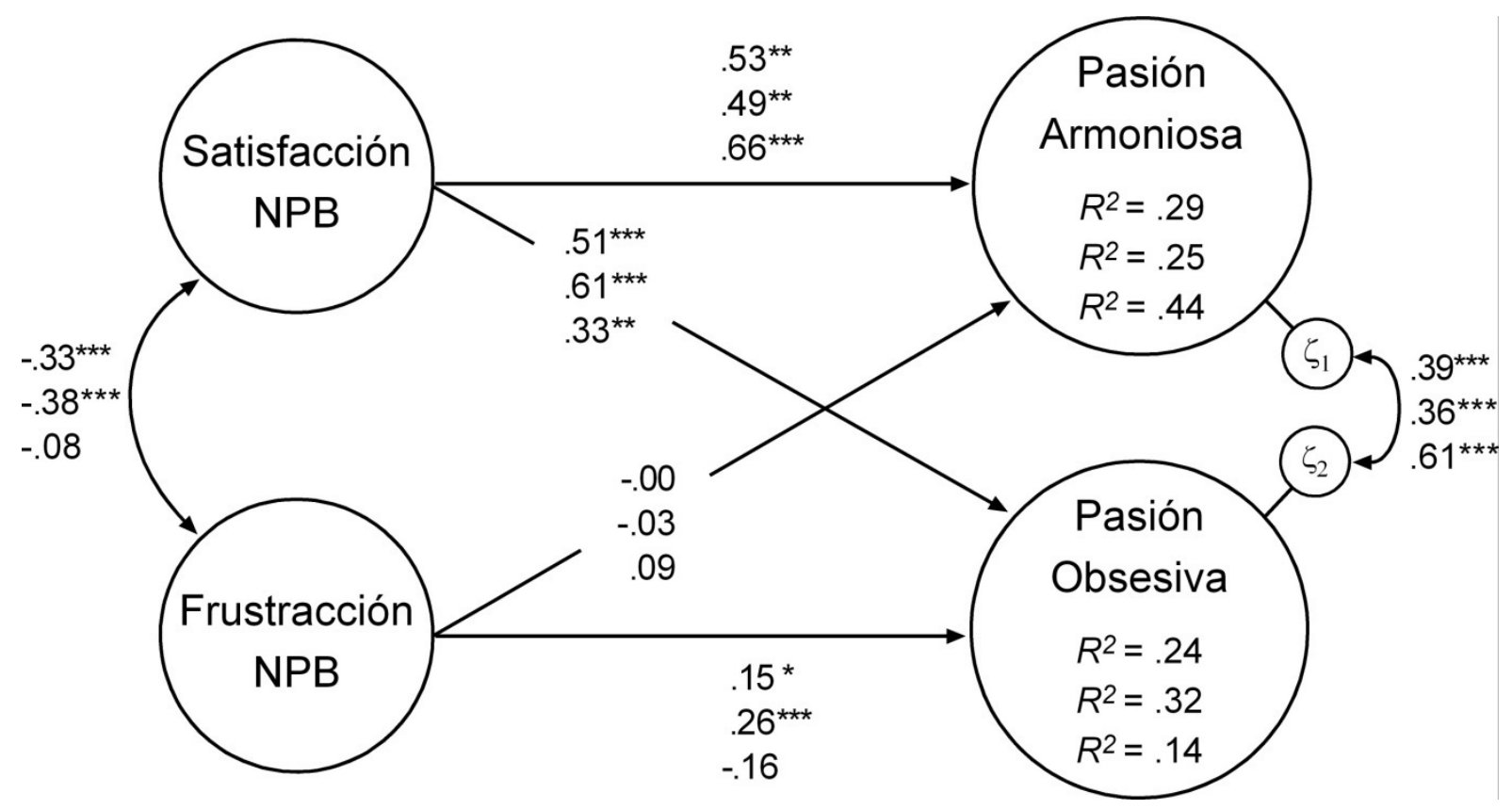

Nota. Se muestran los valores estandarizados para la muestra total (línea superior), hombres (línea central), y mujeres (línea inferior). Los valores de significación mostrados $(p)$ se derivan de los IC al 95\% obtenidos mediante la técnica de bootstrapping de 5000 muestreos.

${ }^{*} p<.05 ; * * p<.01 ; * * * p<.001$.

Figura 2.Modelo de ecuaciones estructurales que muestra la relación entre la satisfacción y la frustración de las necesidades psicológicas básicas y las formas armoniosa y obsesiva de pasión por el ejercicio.

\section{Discusión}

Considerando los postulados teóricos de la TAD y del MDP, el presente estudio analizó los efectos diferenciados de la percepción de satisfacción y frustración de las necesidades psicológicas básicas sobre las formas armoniosa y obsesiva de la pasión en el ejercicio. Los resultados de este estudio apoyan los planteamientos teóricos de la TAD en cuanto al carácter diferenciado de las consecuencias asociadas a la percepción de satisfacción y frustración de las necesidades psicológicas básicas (Deci y Ryan, 2000; Ryan y Deci, 2000). Además, los resultados obtenidos se sitúan en línea con el conjunto de estudios que sugieren el carácter predominantemente desadaptativo asociado a las consecuencias derivadas de la percepción de frustración de las necesidades en el contexto del ejercicio (Bartholomew et al., 2011; Costa et al., 2015; Sicilia et al., 2013). De esta forma, este estudio sugiere la conveniencia de considerar el papel de ambos constructos (i.e., satisfacción y frustración) a la hora de explicar el proceso que determina el carácter armonioso u obsesivo con el que la persona puede llegar a apasionarse por la práctica de ejercicio físico. Por otro lado, los resultados de este estudio se muestran novedosos por cuanto sugieren que la relación entre la percepción de frustración de las necesidades psicológicas básicas y la pasión por el ejercicio podría diferir en función del género.

Respecto a la primera de las hipótesis planteadas, los resultados de este estudio se muestran en línea con estudios previos (Lalande et al., 2015; Parastatidou et al., 2012) y los planteamientos teóricos del MDP, dado que la percepción de satisfacción de las necesidades 
psicológicas básicas experimentada en el contexto del ejercicio predijo positivamente las dos formas de pasión (i.e. armoniosa y obsesiva).Por contra, la segunda de las hipótesis planteadas tan solo fue respaldada de manera parcial, ya que pese a que la frustración de las necesidades psicológicas básicas predijo la PO en sentido positivo en hombres, dicha predicción se produjo en sentido negativo en el caso de las mujeres, aunque de forma muy débil y sin alcanzar el nivel de significación estadística.

El estudio previo de Lalande et al. (2015) mostró que la satisfacción de las necesidades psicológicas básicas experimentada en el contexto del ejercicio físico contribuía en mayor medida al desarrollo de la PA que de la PO, sin embargo, esta investigación no consideró la posible influencia del género en dicha relación. Al igual que en el estudio de Lalande et al., en el presente trabajo la satisfacción de las necesidades psicológicas básicas explicó en mayor medida la PA que la PO en el caso de las mujeres. Sin embargo, en la submuestra formada por hombres se observó el efecto contrario; es decir, la percepción de satisfacción influyó en mayor medida en el desarrollo de la PO que en el de la PA. Estos resultados parecen poner en cuestión la hipótesis planteada por Lalande et al. según la cual la asociación positiva entre la satisfacción de las necesidades psicológicas básicas en el ejercicio y la forma de pasión armoniosa por esta actividad sería más fuerte que la asociación positiva mostrada entre la satisfacción de las necesidades y la forma de pasión obsesiva.

Los resultados de este estudio sugieren más bien que las experiencias personales implícitas en la percepción de satisfacción y frustración de las necesidades psicológicas básicas en el contexto del ejercicio físico podrían influir de manera diferente en la forma de implicarse hombres y mujeres en la conducta del ejercicio. Aunque estos resultados parecen contradecir los postulados de la TAD, que establecen el carácter universal de las consecuencias derivadas de la percepción de satisfacción y frustración de las necesidades psicológicas básicas, lo cierto es que estudios previos ya cuestionaron la homogeneidad de los resultados para hombres y mujeres. En concreto, Weman-Josefsson et al. (2015) observaron que la influencia de la satisfacción de las necesidades psicológicas básicas en el proceso de integración de la regulación de la conducta resultaba moderada por el género de los participantes. Una de las posibles explicaciones al efecto moderador del género observado en este estudio podría plantearse atendiendo a las diferencias personales de carácter relativamente estable que pueden orientar a la persona hacia la búsqueda de determinados incentivos (i.e., los motivos implícitos; Schultheiss y Brunstein, 2010; Schultheiss y Hale, 2007). De hecho, la investigación ha mostrado que motivos implícitos relacionados con la necesidad de competencia (i.e., logro) y de relación (i.e., afiliación) podrían moderar la influencia que la percepción de satisfacción y frustración de las necesidades psicológicas básicas ejercen sobre variables de carácter motivacional, cognitivo y conductual propias del contexto del ejercicio físico (Schüler, Sheldon, y Fröhlich, 2010; Schüler, Wegner, y Knechtle, 2014). De este modo, las diferencias que los estudios han mostrado en los motivos implícitos entre hombres y mujeres (Schultheiss y Brunstein, 2001) pudiera también explicar el efecto diferenciado de la percepción de satisfacción y frustración de sus necesidades psicológicas básicas. No obstante, y dado que el diseño del presente estudio no contempló la medida de los motivos implícitos, esta explicación supone tan solo una posibilidad que debería ser corroborada por futuras investigaciones.

Los resultados de este estudio sugieren, por tanto, que el efecto de la percepción de satisfacción y frustración de las necesidades psicológicas básicas sobre la forma de implicarse 
en el ejercicio físico (i.e., las formas de pasión) podría diferir en función del género. En concreto, los resultados apuntarían a la necesidad de intervenir de forma específica en población masculina al objeto de disminuir la percepción de frustración de las necesidades psicológicas básicas en el contexto del ejercicio físico. De esta manera se podría paliar la incidencia del conjunto de consecuencias negativas que la investigación ha asociado a la PO (Lalande et al., 2015; Méndez-Giménez et al., 2016; Paradis et al., 2013).

A pesar de las evidencias mostradas en este estudio acerca de la incidencia de la percepción de satisfacción y frustración de las necesidades psicológicas básicas sobre las formas de pasión en el contexto del ejercicio, una serie de limitaciones merecen ser destacadas. En primer lugar, aunque este estudio aporta evidencias sobre el papel que el género podría tener a la hora de explicar las relaciones contempladas, estos resultados deben ser considerados con cautela debido al carácter exploratorio del efecto de moderación testado. Además, cabe destacar que el diseño transversal empleado en esta investigación impide generalizar los resultados obtenidos. En segundo lugar, anteriores estudios sugieren que la PO podría disminuir con la edad (Chamarro et al., 2015; Marsh et al., 2013) y que esta variable podría interaccionar con el género afectando los niveles de PO. Sin embargo, en este estudio no se ha contemplado la posible interacción entre la edad y el resto de variables dado que la muestra analizada presentaba un rango de edad limitado. Finalmente, la relación entre las necesidades psicológicas básicas y las formas de pasión se estudió en el contexto del ejercicio físico. No obstante, la investigación ha mostrado que un bajo nivel de la satisfacción de las necesidades psicológicas básicas en el dominio general de la vida puede influir en el desarrollo de la PO en el contexto específico del ejercicio físico (Lalande et al., 2015). Por consiguiente, cabe la posibilidad de que los resultados hubiesen diferido de los obtenidos en este estudio de haberse controlado el efecto de la percepción de satisfacción y frustración en el dominio general de la vida sobre la pasión por el ejercicio.

En resumen, los resultados del presente trabajo muestran la importancia de considerar no solo la percepción de satisfacción sino también la de frustración de las necesidades psicológicas básicas a la hora de explicar el proceso que conduce a la persona a desarrollar la pasión por el ejercicio. No obstante, los resultados sugieren que las experiencias personales presentes en el contexto del ejercicio (i.e., las necesidades psicológicas básicas) podrían contribuir a explicar en mayor medida la PA en mujeres y la PO en hombres. Las evidencias obtenidas sugieren la necesidad de profundizar en el papel moderador del género en la influencia que las experiencias subjetivas experimentadas en el contexto del ejercicio pueden ejercer sobre la forma de apasionarse por esta actividad. En concreto, futuros estudios deberían confirmar el efecto moderador del género en la relación entre la percepción de frustración de las necesidades psicológicas básicas y la PO observada en el presente trabajo.

\section{Agradecimientos}

Este trabajo ha sido realizado al amparo del proyecto de investigación "Imagen corporal y ejercicio en la adolescencia: Un estudio longitudinal” (Ref. DEP2014-57228-R), financiado por el Ministerio de Economía y Competitividad en España. 


\section{Referencias}

Anderson, J. C., y Gerbing, D. W. (1988). Structural equation modeling in practice: A review and recommended two-step approach. Psychological Bulletin, 103(3), 411-423.

Bartholomew, K. J., Ntoumanis, N., Ryan, R. M., y Thøgersen-Ntoumani, C. (2011). Psychological need thwarting in the sport context: Assessing the darker side of athletic experience. Journal of Sport \& Exercise Psychology, 33(1), 75-102.

Bentler, P. M. (1989). EQS structural equations program manual. Los Angeles, CA: BMDP Statistical Software.

Bond, F. W., Hayes, S. C., Baer, R. A., Carpenter, K. M., Guenole, N., Orcutt, H. K., ... Zettle, R. D. (2011). Preliminary psychometric properties of the Acceptance and Action Questionnaire-II: A revised measure of psychological inflexibility and experiential avoidance. Behavior Therapy, 42(4), 676-88.

Browne, M. W., y Cudek, R. (1993). Alternative ways of assessing model fit. En K. A. Bollen \& J. S. Long (Eds.), Testing structural equation models (pp. 136-162). Newbury Park, CA: Sage.

Byrne, B. M. (2013). Structural equation modeling with AMOS: Basic concepts, applications, and programming. New York, NY: Routledge.

Carbonneau, N., Vallerand, R. J., y Massicotte, S. (2010). Is the practice of yoga associated with positive outcomes? The role of passion. The Journal of Positive Psychology, 5(6), 452-465.

Chamarro, A., Penelo, E., Fornieles, A., Oberst, U., Vallerand, R. J., y Fernández-Castro, J. (2015). Psychometric properties of the Spanish version of the Passion Scale. Psicothema, 27(4), 402-409.

Chen, F. F. (2007). Sensitivity of goodness of fit indexes to lack of measurement invariance. Structural Equation Modeling, 14(3), 464-504. doi:10.1080/10705510701301834

Costa, S., Coppolino, P., y Oliva, P. (2015). Exercise dependence and maladaptive perfectionism: The mediating role of basic psychological needs. International Journal of Mental Health and Addiction, 1-16.

Deci, E. L., y Ryan, R. M. (2000). The "what" and "why" of goal pursuits: Human needs and the self-determination of behavior. Psychological Inquiry, 11(4), 227-268.

Eime, R. M., Young, J. A., Harvey, J. T., Charity, M. J., y Payne, W. R. (2013). A systematic review of the psychological and social benefits of participation in sport for adults: informing development of a conceptual model of health through sport. International Journal of Behavioral Nutrition and Physical Activity, 10(1), 135-148.

Fleiss, J. L. (2011). Design and analysis of clinical experiments. New York, NY: John Wiley \& Sons.

Geldhof, G. J., Preacher, K. J., y Zyphur, M. J. (2014). Reliability estimation in a multilevel confirmatory factor analysis framework. Psychological Methods, 19(1), 72-91.

Hair, J. F., Black, W. C., Babin, B. J., Anderson, R. E., y Tatham, R. L. (2006). Multivariate data analysis (7th ed.). Upper Saddle River, NJ: Pearson Prentice Hall.

Hambleton, R. K. (1996). Adaptación de tests para su uso en diferentes idiomas y culturas: fuentes de error, posibles soluciones y directrices prácticas. En J. Muñiz (Ed.), Psicometría (pp. 207 - 238). Madrid: Universitas. 
Hötting, K., y Röder, B. (2013). Beneficial effects of physical exercise on neuroplasticity and cognition. Neuroscience and Biobehavioral Reviews, 37, $2243-57$.

Hu, L., y Bentler, P. M. (1999). Cutoff criteria for fit indexes in covariance structure analysis: Conventional criteria versus new alternatives. Structural Equation Modeling: A Multidisciplinary Journal, 6(1), 1-55.

Lalande, D., Vallerand, R. J., Lafrenière, M.-A. K., Verner-Filion, J., Laurent, F.-A., Forest, J., y Paquet, Y. (2015). Obsessive Passion: A compensatory response to unsatisfied needs. Journal of Personality, 2-31.

Marsh, H. W., Hau, K.-T., y Zhonglin, W. (2004). In search of golden rules: Comment on hypothesis-testing approaches to setting cutoff values for fit indexes and dangers in overgeneralizing Hu and Bentler's (1999) findings. Structural Equation Modeling, 11(3), 320-341.

Marsh, H. W., Vallerand, R. J., Lafrenière, M.-A. K., Parker, P., Morin, A. J. S., Carbonneau, N., \& Paquet, Y. (2013). Passion: Does one scale fit all? Construct validity of two-factor passion scale and psychometric invariance over different activities and languages. Psychological Assessment, 25(3), 796-809.

Méndez-Giménez, A., Cecchini, J. A., y Fernández-Río, J. (2016). Pasión por el deporte, actividad física vigorosa y satisfacción con la vida. Revista de Psicología Del Deporte, 25(1).

Milfont, T. L., y Fischer, R. (2010). Testing measurement invariance across groups: Applications in cross-cultural research. International Journal of Psychological Research, 3, 111-121.

Paradis, K. F., Cooke, L. M., Martin, L. J., y Hall, C. R. (2013). Too much of a good thing? Examining the relationship between passion for exercise and exercise dependence. Psychology of Sport \& Exercise, 14(4), 493-500.

Parastatidou, I. S., Doganis, G., Theodorakis, Y., y Vlachopoulos, S. P. (2012). Exercising with passion: Initial validation of the Passion Scale in exercise. Measurement in Physical Education and Exercise Science, 16(2), 119-134.

Raykov, T. (2004). Behavioral scale reliability and measurement invariance evaluation using latent variable modeling. Behavior Therapy, 35, 299-331.

Rousseau, F. L., y Vallerand, R. J. (2008). An examination of the relationship between passion and subjective well-being in older adults. The International Journal of Aging and Human Development, 66(3), 195-211.

Ryan, R. M., y Deci, E. L. (2000). The darker and brighter sides of human existence: Basic psychological needs as a unifying concept. Psychological Inquiry, 11(4), 319-338.

Sánchez, J. M., y Núñez Alonso, J. L. (2007). Análisis preliminar de las propiedades psicométricas de la versión española de la Escala de Necesidades Psicológicas Básicas en el Ejercicio Físico. Revista Iberoamericana de Psicología Del Ejercicio Y El Deporte, 2(2), 83-92.

Schüler, J., Sheldon, K. M., y Fröhlich, S. M. (2010). Implicit need for achievement moderates the relationship between competence need satisfaction and subsequent motivation. Journal of Research in Personality, 44(1), 1-12.

Schüler, J., Wegner, M., y Knechtle, B. (2014). Implicit motives and basic need satisfaction in extreme endurance sports. Journal of Sport \& Exercise Psychology, 36(3), 293-302. 
Schultheiss, O. C., y Brunstein, J. C. (2001). Assessment of implicit motives with a research version of the TAT: picture profiles, gender differences, and relations to other personality measures. Journal of Personality Assessment, 77(1), 71-86.

Schultheiss, O. C., y Brunstein, J. C. (2010). Implicit motives. Oxford University Press.

Schultheiss, O. C., y Hale, J. A. (2007). Implicit motives modulate attentional orienting to facial expressions of emotion. Motivation and Emotion, 31(1), 13-24.

Sicilia, A., Ferriz, R., y Sáenz-Álvarez, P. (2013). Validación española de la Escala de Frustración de las Necesidades Psicológicas (EFNP) en el ejercicio físico. Psychology, Society, \& Education, 5(1), 1-19.

Stebbings, J., Taylor, I. M., Spray, C. M., y Ntoumanis, N. (2012). Antecedents of perceived coach interpersonal behaviors: the coaching environment and coach psychological welland ill-being. Journal of Sport \& Exercise Psychology, 34(4), 481-502.

Stephan, Y., Deroche, T., Brewer, B. W., Caudroit, J., y Le Scanff, C. (2009). Predictors perceived susceptibility to sport-related injury among competitive runners: The role of previous experience, neuroticism, and passion for running. Applied Psychology, 58(4), $672-687$.

Vallerand, R. J. (2010). On passion for life activities: The dualistic model of passion. En M. P. Zanna (Ed.), Advances in Experimental Social Psychology (Vol. 42, pp. 97-193). New York, NY: Academic Press.

Vallerand, R. J. (2012). Passion for sport and exercise: The Dualistic Model of Passion. En G. Roberts \& D. Treasure (Eds.), Advances in motivation in sport and exercise. Champaign, IL: Human Kinetics.

Vallerand, R. J., Blanchard, C., Mageau, G. A., Koestner, R., Ratelle, C., Leonard, M., \& Marsolais, J. (2003). Les passions de l'ame: On obsessive and harmonious passion. Journal of Personality and Social Psychology, 85(4), 756-767.

Vallerand, R. J., Rousseau, F. L., Grouzet, F. M. E., Dumais, A., Grenier, S., y Blanchard, C. M. (2006). Passion in sport: A look at determinants and affective experiences. Journal of Sport and Exercise Psychology, 28(4), 454-478.

Vlachopoulos, S. P., y Michailidou, S. (2006). Development and initial validation of a measure of autonomy, competence, and relatedness in exercise: The Basic Psychological Needs in Exercise Scale. Measurement in Physical Education and Exercise Science, 10(3), 179_ 201.

Weman-Josefsson, K., Lindwall, M., y Ivarsson, A. (2015). Need satisfaction, motivational regulations and exercise: Moderation and mediation effects. The International Journal of Behavioral Nutrition and Physical Activity, 12(1), 67. 